\title{
La digitalización de ambientes de aprendizaje: reto impostergable
}

\author{
ANA AURORA VIDAL-MARTÍNEZ ${ }^{a}$, \\ BEATRIZ OLIVIA CAMARENA-GÓMEZ ${ }^{b}$
}

RESUMEN Hoy en día, es común que las instituciones educativas utilicen tecnologías a fin de enriquecer los ambientes de aprendizaje. Sin embargo, son pocos los estudios sistemáticos que analizan el impacto de tales procesos de cambio en los objetivos de los programas académicos. La información que presenta este documento pretende contribuir a tal análisis. Para el proceso de digitalización del programa académico se utilizaron la plataforma Moodle (PM) y el sistema de videoconferencia Blue Jeans (VBJ). La PM se eligió por su fácil manejo, y el VBı con el propósito de grabar las sesiones del curso y facilitar así los enlaces a distancia entre los participantes. El proceso de monitoreo y seguimiento de esta transformación digital se realizó durante todo el semestre a través de una orientación teórica tanto en lo relativo a los conceptos centrales de los ambientes de aprendizaje como en lo que concierne al enfoque por competencias y usos de la tecnología en la educación. Se concluye que el proceso de digitalización adoptado logró fortalecer la interacción comunicativa de la comunidad escolar en torno a los objetivos del curso.

PALABRAS CLAVE educación en línea, entorno virtual, estrategias educativas, herramientas virtuales, recursos didácticos.

\section{HISTORIA DEL ARTÍCULO}

¿CÓMO CITAR?:

Vidal-Martínez, A. \& CamarenaGómez, B. (2018).La digitalización de ambientes de aprendizaje: reto impostergable. Perspectiva Empresarial, 5(1), 7-16. http:// dx.doi.org/10.16967/rpe.v5n1a1

RECIBIDO: 11 de octubre de 2017 APROBADO: 06 de febrero de 2018

CORRESPONDENCIA:

Ana Aurora Vidal-Martínez, Carretera a La Victoria km o.6, Hermosillo, Sonora, México, C.P. 83304, México.

a Magíster, investigadora asociada del Centro de Investigación en Alimentación y Desarrollo, A.C. México. Correo electrónico: avidal@ciad.mx

b Doctora, investigadora titular del Centro de Investigación en Alimentación y Desarrollo, A.C. México. Correo electrónico: betica@ciad.mx 


\section{¿CóMO CITO EL ARTÍCULO? HOW TO CITE THIS PAPER?}

CHICAGO:

Vidal-Martínez, Ana y Camarena-Gómez, Beatriz. 2018. "La digitalización de ambientes de aprendizaje: reto impostergable". Perspectiva Empresarial 5(1): 7-16. http:// dx.doi.org/10.16967/rpe. v5n1a1

MLA:

Vidal-Martínez, Ana y Camarena-Gómez, Beatriz. "La digitalización de ambientes de aprendizaje: reto impostergable". Perspectiva Empresarial 5.1 (2018): 7-16. Digital. http://dx.doi. org/10.16967/rpe.v5n1a1

\section{The Digitalization of Learning Environments: A Challenge that Cannot be Put Off}

ABSTRACT Nowadays it is common for educational institutions to use technologies in order to enrich learning environments. However, there are few systematic studies that analyze the impact of such change processes on the objectives of academic programs. The information presented in this document intends to contribute to such analysis. The Moodle platform (MP) and the Blue Jeans videoconferencing (BJV) system were used to digitize the academic program. MP was chosen because it is easy to use, and BJv for recording the course sessions and thus facilitate remote connections among participants. The monitoring and follow-up process of this digital transformation was carried out throughout the semester through theoretical guidance both in relation to the central concepts of learning environments and regarding the competency approach and uses of technology in education. It is concluded that the digitalization process adopted strengthened the communicative interaction of the school community around the course objectives.

KEYWORDS virtual environment, educational strategies, online education, virtual tools, teaching resources.

\section{A digitalização de ambientes de aprendizagem: desafio urgente}

RESUMO Hoje em dia, é comum que as instituições educativas utilizem tecnologias a fim de enriquecer os ambientes de aprendizagem. Porém, são poucos os estudos sistemáticos que analisam o impacto desses processos de transformação nos objetivos dos programas acadêmicos. A informação que apresenta este documento pretende contribuir na realização dessa análise. Para o processo de digitalização do programa acadêmico foi utilizada a plataforma Moodle (PM) e o sistema de videoconferência Blue Jeans (VBJ). A PM foi escolhida pela sua fácil utilização e o VBJ foi escolhido com o propósito de gravar as sessões do curso e disponibilizar, assim, os links à distância entre os participantes. 0 processo de monitoramento e seguimento dessa transformação digital foi realizado durante todo o semestre através de uma orientação teórica, tanto no que diz respeito aos conceitos centrais dos ambientes de aprendizagem quanto no que tange o enfoque por competências e usos da tecnologia na educação. Conclui-se que o processo de digitalização adotado conseguiu fortalecer a interação comunicativa da comunidade escolar em torno dos objetivos do curso.

PALAVRAS CHAVE entorno virtual, estratégias educativas, educação on-line, ferramentas virtuais, recursos didáticos. 


\section{Introducción}

La tecnología es parte de nuestra vida cotidiana. Está presente en el hogar, en el trabajo, en la escuela, en los servicios de salud, en la diversión, en el transporte, etc. En el caso de los sistemas de educación formal es claro que la tecnología se puede utilizar en los ambientes de aprendizaje y potenciar así la posibilidad de avanzar en al menos dos de los pilares básicos de la educación: el aprender a conocer o aprender a aprender, y el aprender a vivir juntos (Delors, 1997). De ahí la importancia de introducir la tecnología en los programas escolares.

No obstante, la mayoría de las instituciones educativas de los países en desarrollo carecen de los recursos suficientes que les permitan integrar las tecnologías en sus respectivos ambientes de aprendizaje. Aun así, se tiene conocimiento de algunas experiencias que han empezado a explorar el uso de recursos abiertos y de redes de colaboración con el fin de fomentar y reforzar las competencias de interés en sus respectivas comunidades escolares. El proyecto universitario español a resaltar es el realizado por la Universidad Politécnica de Madrid (UPM), con el OpenCourseWare (http:// ocw.upm.es/). Este crea un espacio web de acceso libre, gratuito y universal a los recursos educativos como soporte a la enseñanza de sus asignaturas. El proyecto Siyavula produce textos educativos para educación media que se distribuyen de manera libre y gratis en línea, especialmente a través de conexiones celulares (los impresos se distribuyen con fondos públicos). Este proyecto nació con colaboradores desde diferentes partes del mundo; ha contado con estudiantes de doctorado y profesores de Australia y el Reino Unido, profesores y alumnos de universidades de Estados Unidos, así como ingenieros y estudiantes de la Universidad de Ciudad del Cabo (UCT), de la Universidad de Stellenbosch y la Universidad de Rhodes (ESVI-AL, 2013).

Otra experiencia de gran relevancia en la generación de contenidos abiertos es la realizada por alumnos de la Facultad de Ingeniería Eléctrica e Informática de la Universidad de Tecnología y Economía de Budapest, en Hungría, quienes,

Han creado una enorme central de recursos educativos que incluye apuntes de clase, enunciados de exámenes con respuestas modelo y resúmenes de artículos y libros. Sin ninguna ayuda institucional, los alumnos han compilado unas 10.000 páginas de contenidos. Porque se han dado cuenta de que compartir sus apuntes les beneficia a todos, haciendo que el aprendizaje fuera más eficiente y más fácil recuperar las clases a las que no habían podido asistir. (Schmidt, 2007)

El hecho es que en la actual sociedad del conocimiento la digitalización constituye un reto más para los países, en general, y las instituciones educativas en particular: el desarrollo tecnológico está cada vez más imbricado en casi todos los espacios, actividades y servicios. Botero (2012) precisa al respecto:

Los proyectos vinculados con la idea de educación abierta suponen que cuando docentes y estudiantes comparten abiertamente su material educativo, como currículos, talleres, presentaciones, preguntas de exámenes y notas de clase, todos nos beneficiamos. Así resume la autora el pensamiento que tiene sobre la utilidad de los recursos educativos abiertos.

Ahora bien, el uso de las tecnologías de la información y la comunicación (TIC) en los entornos de aprendizaje tiende a recrear ambientes virtuales para facilitar o reforzar la comunicación, el procesamiento, la gestión y la distribución de la información. Rodríguez-Vite (2012) define los ambientes de aprendizaje como las condiciones físicas, sociales y educativas en las que se ubican las situaciones de aprendizaje; el tipo de instalaciones, el equipo, las estrategias, las didácticas y las herramientas tecnológicas, así como el contexto y el clima de las relaciones sociales. De manera que el diseño de nuevos ambientes de aprendizaje permite conceptualizar la forma de aprender y enseñar en conformidad con el desarrollo de la sociedad, al apoyarse en nuevos recursos, como, por ejemplo, las TIC. Esto con la finalidad de optimizar todos los componentes del proceso de aprendizaje-enseñanza, y en procura de la formación del estudiante (Ferreiro \& De Napoli, 2008).

En este sentido, el éxito de los ambientes virtuales — como bien afirma Ordoñez (2013) - , depende de su respectiva planeación y aceptación por parte de las autoridades institucionales que pretenden ponerlos en marcha, así como por los docentes que han aceptado involucrarse en esas nuevas formas de enseñar. Cubiertos estos requisitos, el siguiente paso a considerar cuando se emplean las Tic en el proceso de enseñanza-aprendizaje tiene que ver con identificar las necesidades y las posibilidades reales de su particular empleo 
e integración al contexto particular de aplicación. Asimismo, al diseñar nuevos ambientes de aprendizaje digitalizados se debe considerar que el empleo de las Tic sea acorde con el conocimiento y el potencial del grupo con relación a su uso (sus particulares conocimientos, destrezas y habilidades al respecto). Esto último, sobre todo porque el propósito de tal cambio se vincula con alcanzar una participación e interacción más activa entre los sujetos que conforman la comunidad escolar (estudiante-estudiante, estudiante-maestro), así como la interactividad estudiante-contenido de enseñanza. Sin duda, las actividades planificadas con tales principios permitirán estrechar estas relaciones de colaboración, de modo que la función del docente será facilitar y mediar en esas posibles interacciones y esos procesos educativos (Ferreiro, 2003).

Sirva lo anterior como breve introducción y presentar así la experiencia de digitalización que se llevó a cabo en el ciclo escolar 2015-II, con el fin de reforzar el diálogo y la reflexión interactiva de los participantes en torno al propósito educativo del curso en y entre los estudiantes de una comunidad escolar. Se trata de un ambiente de aprendizaje enriquecido con las herramientas de tecnologías de información y comunicación disponibles en el centro escolar objetivo. El ambiente de aprendizaje se construyó con base en una plataforma de libre acceso y un software licenciado del sistema Blue Jeans, ambos susceptibles de emplearse en los sistemas escolares. Este software y la plataforma tecnológica constituyeron la herramienta base para facilitar la participación activa y el trabajo colaborativo; sobre todo se diseñaron actividades con la finalidad de fomentar una frecuente interacción estudiante-estudiante y estudiantes-docente, así como su conexión con el mundo real.

\section{Marco teórico}

Los ambientes o entornos de aprendizaje son instrumentos de mediación que posibilitan las interacciones entre los sujetos y median la relación de estos con el conocimiento, con el mundo, con los hombres y con ellos mismos. Al respecto, Fernández, Carballos \& Delavaut (2008, pp.137-149) reconocen que el uso de tecnologías en ambientes de aprendizajes estimula el potencial de los estudiantes: "En un marco destinado a promover el aprendizaje a partir de estrategias educativas enriquecidas con tic's que pretenden crear situaciones de aprendizaje que estimulen al máximo las potencialidades de los alumnos".

Ahora bien, según Duarte (2003) un ambiente de aprendizaje va más allá del medio físico, pues su parte medular son las relaciones e interacciones del grupo. A fin de facilitar esto es sumamente importante la organización y la disposición espacial de los estudiantes, así como las relaciones entre los elementos de su estructura: las pautas de comportamiento que allí se desarrollan, el tipo de relaciones que mantienen (personas/objetos), las interacciones entre las personas, los roles que se establecen, los criterios que prevalecen y las actividades que se realizan. En este sentido, Herrera (2006) distingue cuatro elementos base en un ambiente de aprendizaje: (a) el proceso de interacción o comunicación entre sujetos, (b) el grupo de herramientas o medios de interacción, (c) una serie de acciones reguladas relativas a ciertos contenidos, y (d) el entorno o espacio en el que se realizan dichas actividades.

En la experiencia que aquí se describe los elementos considerados para diseñar y emprender el proceso de digitalización de un ambiente de aprendizaje fueron tres: (a) elementos básicos de un ambiente de aprendizaje, (b) modalidades y tipos de ambientes de aprendizaje, y (c) fases del diseño de un ambiente de aprendizaje (Herrera, 2006). A continuación, se proporciona una breve explicación de lo que se entiende por cada uno de ellos.

\section{Elementos básicos de un ambiente de aprendizaje}

En el propósito de cumplir su función profesional, se espera que los docentes posean los conocimientos, las estrategias, las habilidades y las competencias necesarias que les permitan lograr los objetivos del curso. El reto para los maestros es cómo integrar todo ese conjunto de elementos y relacionarlos; además, de manera sistemática, a fin de lograr los objetivos planteados en el curso, de la manera más eficaz y eficiente posible. El mismo reto lo tienen los docentes cuando integran la digitalización como ambiente de aprendizaje, pero ahora los elementos que intervienen y dan forma a tal proceso difieren de los elementos de un ambiente de aprendizaje convencional (presencial).

En los ambientes virtuales de aprendizaje se conjugan elementos que el docente-facilitador 
debe conocer y tener en cuenta al diseñar el curso. En ese campo, Mejía (2016) distingue dos tipos de elementos que intervienen en los ambientes virtuales de aprendizaje: los elementos constitutivos que se refieren a los medios de interacción, los recursos, los factores ambientales y los factores psicológicos; y, por otra parte, los elementos conceptuales que aluden a los aspectos que definen el concepto educativo del ambiente virtual (diseño instruccional y diseño de interfaz). A continuación, se presenta una breve acotación de cada elemento con énfasis en los cambios o especificidades que adoptan los procesos de interacción, así como la relación y la forma de presentación de recursos, entre otros, con respecto a los ambientes de aprendizaje presenciales.

Los elementos constitutivos de los ambientes virtuales de aprendizaje son:

- Medios de interacción. Predomina la interacción escrita (en los ambientes no virtuales es oral).

- Los recursos. Son, sobre todo, digitalizados, bien sean texto o imágenes, o bien hipertexto o multimedia. Mientras que en ambientes no virtuales predominan los recursos impresos en forma de textos o escritos (apuntes, anotaciones en la pizarra o pizarrón). En ambos casos se puede contar con apoyos adicionales como, por ejemplo, bibliotecas, hemerotecas, bibliotecas virtuales, sitios web, libros electrónicos, etc.

- Los factores físicos. Se refiere a la iluminación, la ventilación, la disposición del mobiliario, etc. Sin duda, tales condiciones son importantes pero en ambientes virtuales suelen escapar del control de las instituciones y de los docentes.

- Las relaciones psicológicas. Se median por la computadora a través de la interacción. Las nuevas tecnologías actúan en la mediación cognitiva entre las estructuras mentales de los sujetos que participan en el proyecto educativo. El maestro participa básicamente como facilitador y guía del proceso.

Los elementos conceptuales de los ambientes virtuales de aprendizaje son:

- El diseño instruccional. Implica la forma en que se planea el acto educativo. Expresa, de alguna manera, el concepto que se tiene del aprendizaje y del acto educativo. La definición de objetivos y el diseño de las actividades, la planeación y el uso de estrategias y técnicas didácticas, la evaluación y la retroalimentación son algunos de sus elementos, dependiendo del modelo instruccional adoptado. Al igual que en los ambientes no virtuales, estos elementos exigen conocimiento de parte del docente del contenido a enseñar, así como de herramientas didácticas que se puedan establecer de modo explícito en una serie de actividades a manera de tareas o retos a cumplir por parte del estudiante.

- El diseño de la interfaz. Se refiere a la expresión visual y formal del ambiente virtual. Es el espacio virtual en el que han de coincidir los participantes. Las características visuales y de navegación pueden ser determinantes para una operación adecuada del modelo instruccional. Se conjuga con el diseño instruccional, le da forma a este.

\section{Modalidades y tipos de ambientes de aprendizaje}

Los ambientes de aprendizaje pueden llevarse a cabo tanto en la educación presencial como en la denominada no presencial o virtual. En el texto de la Secretaría de Educación Pública, se mencionan cuatro tipos de ambientes de aprendizaje (Ordóñez, 2013):

- Centrado en quien aprende. Es la atención, se pone en los conocimientos, las habilidades, las actitudes y las creencias del espacio, es decir, la sensibilidad hacia las prácticas culturales de los estudiantes y el efecto que estas tienen sobre el aprendizaje en el salón.

- Centrado en el conocimiento. Son aquellos que ayudan a los estudiantes a convertirse en conocedores al aprender, de tal manera que comprendan y realicen la subsiguiente transferencia y, por tanto, son importantes guías para alcanzar estas metas.

- Centrado en la evaluación. Proporcionan oportunidades de retroalimentación y de revisión, y aseguran que lo evaluado sea congruente con las metas de aprendizaje.

- Centrado en la comunidad. Establecen conexiones entre el ambiente escolar y la comunidad. 


\section{Las fases del diseño de un ambiente de aprendizaje de Herrera (2006)}

La fase la conforman tres etapas secuenciales:

- Fase I. De prescripción, en la que se definen anticipadamente los resultados que se esperan del proceso instruccional.

Partes que la conforman:

- Intenciones educativas (perfil de egreso: habilidades, actitudes, valores).

- Objetivos generales que estarán en concordancia con las intenciones educativas del modelo.

-El análisis curricular.

- Los objetivos particulares que deberán plantearse con la idea de hacer más eficiente la práctica educativa, de tal forma que deberán ser ejecutables para lograrla.

- Fase II. De instrumentación, se refiere a las actividades y a los medios que dan forma y materializan la instrucción.

Partes que la conforman:

-Selección de estrategias y medios instruccionales.

- Diseño de actividades y técnicas didácticas.

-Diseño de la interfaz.

- Fase III. De operación y evaluación, es la puesta en marcha del proceso instruccional.

- La conforman la operación y evaluación, con lo cual podremos constatar y aún más corroborar lo válido del proyecto; si se presentaron fallas también nos brinda la posibilidad de corregir el rumbo.

\section{Metodología. Desarrollo del ambiente de aprendizaje}

Según Duarte (2003), los nuevos ambientes de aprendizaje —como, por ejemplo, los digitales- se instauran en las dinámicas que constituyen los procesos educativos e involucran acciones, experiencias y vivencias por cada uno de los participantes. En tales procesos se ponen en juego actitudes, condiciones materiales y socioafectivas, así como múltiples relaciones con el entorno. Requieren, como toda propuesta educativa, de la infraestructura necesaria para la concreción de los propósitos culturales.

La experiencia que se refiere en este documento se llevó a cabo siguiendo varias fases que van desde la selección de apoyos tecnológicos hasta el diseño del programa, la planeación y la ejecución. Primero, a fin de llevar a cabo la digitalización de nuestro ambiente de aprendizaje se utilizó la plataforma de Moodle de código abierto (Sánchez, 2009), la cual es de fácil acceso y muy cómoda tanto para docentes como para los estudiantes, debido a que permite cargar todo el material de interés (del semestre en este caso). También permite llevar la planeación didáctica, diseñar y cargar innumerables tareas en beneficio de la dinámica del curso, a la vez que permite monitorear y dar seguimiento al progreso académico de cada estudiante. Entre las acciones que posibilita esta plataforma se encuentran: añadir usuarios a los cursos de interés; crear perfiles para cada materia; organizar grupos de discusión y trabajo; agendar un calendario sincronizado con todos los estudiantes; corregir y hacer anotaciones de las tareas de los alumnos (sin necesidad de repeticiones); y manejar las calificaciones de una forma sencilla con escalas personalizadas.

La otra herramienta utilizada fue Blue Jeans, la cual se refiere a una sala virtual en la que cada usuario tiene un número único personal. Este sistema posibilita que el participante pueda conectarse fácilmente y de forma simultánea por medio de video, voz y datos (Blue Jeans, 2017). Esta aplicación se utilizó para realizar las grabaciones de cada sesión a fin de, posteriormente, subirla a la plataforma Moodle, de modo que estuviera disponible las 24 horas de los siete días de la semana, y así los alumnos inscritos en el curso tenían la posibilidad de acceder cuántas veces quisieran a la grabación, despejar dudas y hacer las preguntas necesarias.

Previo al proceso de digitalización del ambiente de aprendizaje de un programa escolar, se presentó la propuesta a las autoridades institucionales en espera de su autorización y apoyo para el manejo del paquete tecnológico de interés. Logrados los permisos, se dio a conocer tal posibilidad de digitalizar los ambientes de aprendizaje a un grupo de maestros. Se les invita a ser parte de tal experiencia y se deja claro que se contaría con el apoyo de personal técnico especializado en manejo de tecnologías durante todo el semestre, bien sea para asesorar o atender preguntas, o bien con el fin de resolver dudas vinculadas a tal proceso, 
tanto de parte del docente como de los estudiantes. A continuación se presentan los resultados de esta experiencia.

\section{Principales resultados}

El ambiente de aprendizaje que se apoyó con el uso de las tecnologías de información y comunicación se relaciona con un curso que se imparte en el primer semestre de un programa de posgrado en un centro SEP-Conacyt, ubicado en el noroeste de México. En el proceso de digitalización de tal curso se consideraron varios elementos: las competencias a alcanzar, el nivel de desarrollo de las competencias, el resultado del aprendizaje, las estrategias de enseñanza y aprendizaje y la evaluación. Cada uno de estos elementos lo revisó y ajustó el docente responsable y el responsable de planeación y desarrollo de los programas de posgrado, de manera que se explicitaron de la siguiente forma.

\section{Objetivo general del curso}

A partir de identificar aquellas tradiciones filosóficas que proponen una explicación de los orígenes del conocimiento y la ciencia a través de la historia de la filosofía, el estudiante reflexionará acerca del propósito de la investigación en la actualidad.

\section{Competencias}

Se busca que el estudiante categorice y relacione tanto la evolución del saber como los conceptos, los representantes filosóficos y sus diversas contribuciones al desarrollo civilizatorio.

Se identificarán conceptos particulares determinantes en la investigación científica con el propósito de desarrollar una actitud crítica, histórica y actualizada hacia el proceso de investigación, sus métodos y sus resultados.

\section{Aprendizajes}

Se pretende que el estudiante practique un ejercicio sistemático de análisis crítico de la investigación científica y de su propio quehacer mediante el aprendizaje de conceptos ordenadores, así como el conocimiento de aquellos paradigmas y sistemas teóricos que son determinantes en la construcción de la ciencia en tanto campo especializado, bien sea como un saber específico, o bien como una ética y una práctica contemporánea.

\section{Horas-clase}

Las horas de clase programadas por semana son en total dos.

\section{Estrategias didácticas}

El curso se basó en el desarrollo de lecturas y diálogos aplicados, en los cuales se involucra la investigación científica. Algunos materiales bibliográficos presentados fueron de lectura obligatoria y otros complementarios, con el propósito de reforzar o ampliar la información y los objetivos del curso. El propósito de los materiales seleccionados fue orientar a los estudiantes hacia el descubrimiento de la estrecha relación entre el quehacer cotidiano del hombre y la forma en que se explica y opera en el mundo. El curso constituye un espacio privilegiado de reflexión y formación integral, de modo que se espera del estudiante la práctica de un ejercicio sistemático de análisis crítico de la investigación científica y de su propio quehacer mediante el aprendizaje de conceptos ordenadores. Asimismo, que conozca paradigmas y sistemas teóricos determinantes de la construcción de la ciencia en tanto campo especializado, bien sea como un saber específico, o bien como una ética y una práctica contemporánea.

\section{Actividades de desarrollo}

El docente presentó a los estudiantes la liga de interés al curso (página web). En dicha página-web se encuentran explícitos los contenidos educativos virtuales de la asignatura. El requisito a cumplir para tener acceso a esa liga fue estar inscrito en el curso y registrarse en la plataforma. En cada sesión el docente abordaba los temas correspondientes. En la dinámica programada también había exposiciones de parte de los estudiantes por asignación libre de temas. Cuando se presenta esta última dinámica, el compromiso o responsabilidad del resto de estudiantes que no exponía era escribir un reporte de cada exposición de sus compañeros. Así, quedó establecida con claridad la fecha límite en que se debería subir a la plataforma (página web) el reporte elaborado (uno por tema expuesto). Se acordó que un día antes de la

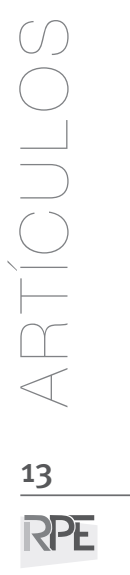


siguiente clase sería la fecha límite para subir el reporte del tema visto en la clase anterior.

Es importante precisar que cada clase generaba un archivo que incluye voz, video y datos, archivo que se subía a la plataforma. El propósito de tal archivo era ayudar a los estudiantes a repasar lo visto en clase o, si por algún motivo no pudo asistir, pudiera consultar lo visto en clase a través de la página web (clase grabada).

Otra ventaja de este tipo de cursos tiene que ver con la plataforma que se usa, toda vez que ofrece una colección de herramientas de diversas características con el propósito de facilitar el proceso de enseñanza-aprendizaje. Las principales herramientas utilizadas en el curso que se describe fueron los foros de dudas y los foros de discusión. Los primeros fueron programados de acceso abierto durante todo el curso con el objetivo de que el estudiante contase con un espacio para exponer sus dudas referentes a la clase y a los contenidos. Las respuestas a tales dudas las brinda directamente el docente y son visibles para todos los alumnos inscritos y registrados en el curso. El otro tipo de foro (de discusión) se utilizó para actividades o temas específicos y con vigencia de participación. En este caso, se trata casi siempre de ofrecer puntos de vista de algún tema o lectura con la cual tanto docentes como alumnos pueden realizar retroalimentación al resto de participaciones.

\section{Recursos y materiales}

Para el estudiante: computadora, Internet, proyector y pantalla.

De la institución/docente: aula equipada con equipo de videoconferencia, plataforma Moodle, sistema Blue Jeans, computadora, Internet, proyector y pantalla.

\section{Evaluación}

Esta fase se lleva a cabo con varios elementos que facilita la plataforma digital. Entre otros, se solicita al estudiante la elaboración de reportes de lectura (con fecha límite para subir a la plataforma y así posibilite la evaluación por parte del docente); exposición individual y en equipo (en clase) de tema asignado por el maestro; elaboración de ensayo; participación en foros y examen escrito.

Por último, a fin de conocer la opinión de los estudiantes con respecto a la experiencia de digitalización del ambiente de aprendizaje en el que participaron, se les solicitó una evaluación del curso en algunas áreas. El rango de calificación sugerido fue de 0 a 10 , en el que 0 es el mínimo y 10 el máximo puntaje. En tal proceso participaron 49 estudiantes y la calificación media obtenida se presenta en la tabla 1.

TABLA 1. Resultados de la experiencia de digitalización de un ambiente de aprendizaje

\begin{tabular}{|c|c|}
\hline CONCEPTO & $\begin{array}{l}\text { CALIFICACIÓN } \\
(\mathrm{N}=49) \\
\text { MEDIA }\end{array}$ \\
\hline Enseñanza & 8,72 \\
\hline $\begin{array}{l}\text { El profesor se preocupó por el } \\
\text { aprendizaje de los alumnos. }\end{array}$ & 8,73 \\
\hline $\begin{array}{l}\text { El profesor transmitió la enseñanza con } \\
\text { claridad. }\end{array}$ & 8,73 \\
\hline $\begin{array}{l}\text { Las evaluaciones realizadas fueron } \\
\text { acordes con los objetivos del curso. }\end{array}$ & 8,70 \\
\hline Competencias & 8,68 \\
\hline $\begin{array}{l}\text { Se presentaron las competencias a } \\
\text { desarrollar al inicio del curso. }\end{array}$ & 8,68 \\
\hline $\begin{array}{l}\text { Las actividades promueven el logro } \\
\text { de las competencias planeadas. }\end{array}$ & 8,68 \\
\hline $\begin{array}{l}\text { Materiales didácticos y habilidades } \\
\text { docentes }\end{array}$ & 8,68 \\
\hline $\begin{array}{l}\text { Las presentaciones del profesor fueron } \\
\text { suficientes y bien estructuradas. }\end{array}$ & 8,68 \\
\hline $\begin{array}{l}\text { Adecuada presentación y acceso a } \\
\text { contenidos del curso. }\end{array}$ & 8,68 \\
\hline Motivación & 8,72 \\
\hline $\begin{array}{l}\text { El profesor logró que el alumno } \\
\text { estudiara con gusto y convicción. }\end{array}$ & 8,68 \\
\hline $\begin{array}{l}\text { El profesor estimuló la crítica } \\
\text { constructiva. }\end{array}$ & 8,72 \\
\hline $\begin{array}{l}\text { El profesor convenció al alumno de la } \\
\text { importancia del curso. }\end{array}$ & 8,76 \\
\hline Opinión global & 8,70 \\
\hline
\end{tabular}

Resultados digitalización de un ambiente de aprendizaje. Posgrado Maestría en CienciasCentro SEP-CONACYT, México.

\section{Conclusiones}

Al igual que a nivel global y nacional, el tipo de experiencia de digitalización de un ambiente de aprendizaje como el descrito en este trabajo marca el inicio de una nueva forma de enseñanza-aprendizaje que llegó para quedarse. El reto es cómo acceder y hacer realidad los ambientes virtuales con el fin de reforzar procesos de enseñanza-aprendizaje en las instituciones educativas 
del país. Sin duda, habrá que mejorar de manera significativa las condiciones físicas, sociales y educativas en las que se ubican o promueven tales situaciones de aprendizaje: el tipo de instalaciones, el equipo, las estrategias, las didácticas, las herramientas tecnológicas y las relaciones sociales (el contexto).

El diseño de nuevos ambientes de aprendizaje permite conceptualizar la forma de aprender y enseñar en concordancia con el desarrollo de la sociedad, al apoyarse en nuevos recursos como son la Tic. Con la finalidad de optimizar todos los componentes del proceso de aprendizaje-enseñanza y en procura de la formación del estudiante (Ferreiro \& De Napoli, 2008).

Las nuevas generaciones de alumnos demandan de manera diferente, sobre todo en el contexto tecnológico. Su atención se enfoca cada vez más en lo visual, lo cual exige a los maestros seleccionar de manera más adecuada las herramientas que ayuden a planear y diseñar ambientes de aprendizaje más atractivos y acordes con los objetivos, las demandas y las necesidades de los estudiantes del siglo XXI.

La experiencia descrita en este documento permite visualizar las siguientes ventajas en los ambientes de aprendizaje basados en competencias y el empleo de TIC:

- El docente, al planear cada sesión de trabajo y diseñar el ambiente de aprendizaje, optimiza el tiempo hora-clase; también visualiza mejor el logro de los objetivos, así como el cumplimiento de la competencia.

- El uso de herramientas tecnológicas permite llevar al estudiante al plano visual y auditivo, así como experimentar sin salir del aula de clase.

- Utilizar herramientas tecnológicas es de bajo costo, fácil acceso y uso (para estudiantes y docentes).

- Utilizar herramientas tecnológicas facilita el aprendizaje y requiere inversión de tiempo de parte del docente, pero este se recupera en clase vía una mejor planeación y con mejores resultados del proceso enseñanza-aprendizaje.

- El uso de plataformas y el trabajo vía remota fortalece el trabajo cooperativo, permite compartir información, emitir juicios, debatir puntos de vista y facilita la comunicación. Esto permite lograr que la separación geográfica no sea una limitante entre estudiante-docentes y estudiantes-estudiante, a fin de lograr el diálogo y la interacción comunicativa.

Los ambientes digitales de aprendizaje lideran los procesos en los países desarrollados y poco a poco ganarán presencia en los demás países del mundo (México entre ellos). En este último país, hace ya dos décadas que se tienen experiencias en ese sentido, principalmente en educación básica (programa de uso de TIC, salón de medios audiovisuales, pizarrón digital; etc.). Sin embargo, en las instituciones de educación superior que ofrecen programas de posgrado - que en su mayoría cuentan con las instalaciones y el equipo tecnológico actualizado- se resisten a realizar tal cambio. La tradición de la enseñanza oral es más fuerte y los docentes se resisten a transitar hacia ambientes digitales de enseñanza-aprendizaje. Esto aun cuando conocer una diversidad de herramientas les puede servir de apoyo para realizar mejor su trabajo. El hecho es que el uso de las TIC se convierte en una ventaja en ese sentido. El hecho de que las nuevas prácticas educativas estuviesen sustentadas por docentes diseñadores de estos espacios virtuales y, sobre todo, que se lograra su vinculación efectiva con las actividades del salón de clases, trascendió en gran medida el proceso de digitalización de ambientes de aprendizaje. Actualmente, en la sociedad existe una brecha digital entre los sectores menos favorecidos; aun con los avances tecnológicos, para muchos estudiantes es difícil tener acceso a las TIC en todo momento, de manera que se presenta la oportunidad de implementar las competencias docentes y hacer frente a esta situación. Este cambio es necesario en el propósito de que la educación de nuestro país dé un paso adelante hacia la calidad educativa.

\section{REFERENCIAS}

Blue Jeans (2017). Getting started guide.

Botero, C. (2012, febrero 16). Arquitectura en proyectos de educación abierta. ElEspectador.com. Recuperado de http://www.elespectador.com/ impreso/opinion/ columna-326993-arquitectura-proyectos-de-educacion-abierta

Delors, J. (1997). La educación encierra un tesoro. Francia: Unesco.

Duarte, J. (2003). Ambientes de aprendizaje una aproximación conceptual. Revista Iberoamericana de 
Educación. Recuperado de http://www.rieoei.org/ deloslectores/524Duarte.PDF

Educación Superior Virtual Inclusiva-América Latina-ESVI-AL. (2013). Informe de estado del arte de recursos educativos abiertos que puedan apoyar la formación superior virtual de personas con discapacidad. Recuperado de http://www.esvial.org/ wp-content/files/E114_vf_v4.pdf

Fernández, R. R., Carballos, E. \& Delavaut, M. (2008). Un modelo de autoaprendizaje con integración de las TIC y los métodos de gestión del conocimiento. Revista Iberoamericana de Educación a Distancia, 11(2), 137-149. Recuperado de http://www. biblioteca.org.ar/libros/141704.pdf

Ferreiro, R. (2003). Estrategias didácticas del aprendizaje cooperativo. Una nueva forma de aprender y enseñar: El constructivismo. México: Editorial Trillas.

Ferreiro, R. \& De Napoly, A. (2008). Más allá del salón de clases: los nuevos ambientes de aprendizajes. Revista Complutense de Educación, 19(2), 333,346. Recuperado de: https://revistas.ucm. es/index.php/RCED/article/viewFile/ RCED0808220333A/15480

Herrera, M. Á. (2006). Consideraciones para el diseño didáctico de ambientes virtuales de aprendizaje: una propuesta basada en las funciones cognitivas del aprendizaje. Revista Iberoamericana de Educación, 38(5). Recuperado de http://www.rieoei. org/deloslectores/1326Herrera.pdf

Mejía, M. P. (2016). Diseño y desarrollo de Learning Objects, integrados en un aula virtual, enfocados al aprendizaje de la contabilidad general. Riobamba: Escuela Superior Politécnica de Chimborazo. Recuperado de http://dspace.espoch.edu.ec/handle/123456789/4739\#sthash.5HiBhVDz.dpuf

Ordóñez, M. (2013). Análisis y diseño de un ambiente de aprendizaje enriquecido con el uso de las tecnologías bajo el enfoque por competencias sea para el ámbito académico o laboral. Planeación y evaluación educativa, 19(57), 13-24. Recuperado de http://www.aragon.unam.mx/unam/difusion/ planeacion/ejemplares/57.pdf

Sánchez, I. J. (2009). Plataforma educativa Moodle: administración y gestión. México: Alfaomega/Ra-Ma.

Schmidt P. (2007). Recursos educativos abiertos: estrategia para apertura y desarrollo social de la educación superior.

Rodriguez-Vite, H. (2012). Ambientes de aprendizaje. Universidad Autónoma el Estado de Hidalgo. Recuperado de http://www.uaeh.edu.mx/scige/ boletin/huejutla/n4/e1.html 